\title{
THE MOMENTS OF CERTAIN COMPLEX POTENTIALS
}

\author{
HOWARD D. FEGAN AND PETER B. GILKEY ${ }^{1}$
}

\begin{abstract}
Let $M$ be a compact homogeneous space, let $\Delta$ be the Laplacian, and let $V$ be the vector space of Fegan potentials. If $q \in V$, then $\Delta$ and $\Delta+q$ have the same spectrum. We show that all the moments of such a potential must vanish.
\end{abstract}

1. Introduction. Let $M$ be a homogeneous space $G / H$, where $G$ and $H$ are compact Lie groups. Let $\Delta$ be the Laplace-Beltrami operator on $M$. We choose the sign so the eigenvalues of $\Delta$ are all nonnegative. Since $G$ is compact, it acts naturally on $L^{2}(M)$. We decompose this representation into a sum of irreducible representations

$$
L^{2}(M)=\sum_{\lambda} H_{\lambda}
$$

Choose a highest weight vector $v_{\lambda}$ in each $H_{\lambda}$. Let $V \subset L^{2}(M)$ denote the complex vector space generated by all the $v_{\lambda}$. If $q \in V$, then $q$ is isospectral to the zero potential

$$
\operatorname{spec}(\Delta)=\operatorname{spec}(\Delta+q) \quad \forall q \in V .
$$

For example, if $M=S^{1}$ is the circle, then

$$
V=\left\{q=\sum_{n>0} a_{n} e^{i n \cdot \theta}\right\}
$$

are the Hardy functions given by a Fourier series involving positive integers $n$ only. We refer to $[2]$ for further details. These complex-valued functions form a real vector space. This generates many isospectral deformations.

This raises two problems: the inverse problem, and the problem of describing some of the properties of these potentials. In this note we prove

THEOREM 1.1. Let $M$ be a compact Riemannian manifold without boundary. Let $\Delta$ denote the Laplacian on $M$. Let $q$ be a potential for $\Delta$ and assume that the spectra of $\Delta$ and of $\Delta+r \cdot q$ agree for at least $k+1$ distinct values of $r \in R$. Then

$$
\int_{M} q^{j}=0 \quad \text { for } j \leq k
$$

THEOREM 1.2. Let $M$ be a compact Riemannian manifold without boundary. Let $\Delta$ denote the Laplacian on $M$. Let $V$ be a real vector space. Assume that $\Delta$ and $\Delta+q$ have the same spectrum for all $q \in V$. Let $\left\{q_{1}, \ldots, q_{k}\right\} \subset V$. Then

$$
\int_{M} q_{1} \cdots q_{k}=0
$$

Received by the editors April 12, 1985.

1980 Mathematics Subject Classification. Primary 58G25; Secondary 58G11.

${ }^{1}$ Research partially supported by NSF grant MCS-8301896. 
REMARK. In fact these two results continue to hold if $\Delta$ is replaced by a selfadjoint elliptic differential operator with positive definite leading symbol acting on a complex line bundle over $M$. The vector-valued case is quite different, and these methods do not work.

REMARK. The Fegan potentials satisfy the hypotheses of both theorems, and thus this describes some of the properties of the known examples. In particular, all the moments of the Fegan potentials vanish.

The Laplacian $\Delta$ is a real differential operator. Consequently $\bar{V}=\{\bar{q}: q \in V\}$ also satisfies the hypotheses of Theorem 1.2.

COROLlarY 1.3. Let $V$ satisfy the hypotheses of Theorem 1.2. Then $V \cap \bar{V}=$ $\{0\}$.

REMARK. In particular, none of the Fegan potentials can be real.

ProOF. Let $q \in V \cap \bar{V}$. Then by Theorem 1.1, $\int_{M} q^{2}=0$, which implies that $q=0$ since $q$ is real.

In Theorem 1.1, we assume the isospectral property holds for many values of the real parameter $r$. We do not know of any examples in which $\operatorname{spec}(\Delta)=\operatorname{spec}(\Delta+q)$ holds without $\operatorname{spec}(\Delta)=\operatorname{spec}(\Delta+r q)$ holding for all $r$. If one passes to first-order operators, however, such a condition is easily satisfied;

$$
\operatorname{spec}(i \partial / \partial \theta)=\operatorname{spec}(i \partial / \partial \theta+r)
$$

holds for all $r \in Z$ but not for all $r \in R$.

2. Proof of the theorems. Let $M$ be a compact Riemannian manifold of dimension $m$ without boundary. We recall the spectral invariants for the operator $P=\Delta+q$ on $M$. Let $Z(t, P)$ denote the trace of the heat kernel so that

$$
Z(t, P)=\sum_{i} e^{-t \lambda_{i}}
$$

where the sum is over the eigenvalues of $P$. Then as $t \rightarrow 0^{+}$there is an asymptotic expansion of the form

$$
Z(t, p) \approx(4 \pi t)^{-m / 2} \sum_{n=0}^{\infty} a_{n}(P) t^{n}
$$

The $a_{n}(P)$ are local invariants of the operator $P$. In particular, if we let $P_{r}=\Delta+r \cdot q$ for $r \in R$, then

$$
a_{n}\left(P_{r}\right)=\sum_{j=0}^{n} a_{n, j}(\Delta, q) r^{j} .
$$

The leading term is particularly easy to compute, and in $[\mathbf{1}]$ it was shown that

$$
a_{n, n}(\Delta, q)=\frac{1}{n !} \int_{M} q^{n}
$$

where the integral is with respect to the Riemannian element of volume defined on $M$. It is immediate from the definition that the $a_{n, j}$ are spectral invariants. Let $\Delta$ and $\Delta+r \cdot q$ have the same spectrum for at least $n+1$ values of $r$. Since $a_{n}\left(P_{r}\right)=a_{n}(P)$ is a polynomial of degree at most $n$ in $r$, we conclude that $a_{n}\left(P_{r}\right)$ 
is independent of $r$ and, therefore, that $a_{n, n}(\Delta, q)$ is zero. By equation (2.4) this is exactly the content of Theorem 1.1 and completes the proof.

Next suppose that $V$ is a real vector space of isospectral potentials. Let $r=$ $\left(r_{1}, \ldots, r_{k}\right)$ and let $q(r)=r_{1} \cdot q_{1}+\cdots+t_{k} \cdot q_{k}$, where the $q_{i} \in V$. Let $I=\left(i_{1}, \ldots, i_{k}\right)$ be a multi-index and adopt the usual multi-index notation. We polarize to see

$$
\int_{M} q(r)^{k}=\sum_{|I|=k} r^{I} \int_{M} q^{I}
$$

where $r^{I}=\prod_{\nu} r_{\nu}^{i_{\nu}}$ and $q^{I}=\prod_{\nu} q_{\nu}^{i_{\nu}}$. By Theorem 1.1 this vanishes identically in $r$, and consequently all the coefficients vanish identically. This proves Theorem 1.2.

\section{REFERENCES}

1. P. Gilkey, Recursion relations and the asymptotic behavior of the eigenvalues of the Laplacian, Compositio Math. 38 (1979), 201-240.

2. V. Guillemin and A. Uribe, Spectral properties of a certain class of complex potentials, Trans. Amer. Math. Soc. 279 (1983), 759-771.

Department of Mathematics, University of New Mexico, Albuquerque, NeW MEXICO 87131

Department of MAThematics, UNIVERsity of OREgon, EugENE, OREgon 97403 